\title{
Eccrine hidradenoma of the breast: distinct pathological lesion mimicking a carcinoma
}

\section{Olfa El Amine El Hadj'1, Marwa Mhiri', Aida Goucha', Jameleddine Ben Hassouna², Olfa Adouni' ${ }^{1}$, Khaled Rahal' ${ }^{2}$,Ahmed El May', Amor Gamoudi'}

${ }^{1}$ Department of Pathology, Salah Azaiez Institute, Tunis, Tunisia, ${ }^{2}$ Department of Carcinological Surgery, Salah Azaiez Institute, Tunis, Tunisia

Corresponding author: Dr. Olfa El Amine El Hadj, E-mail: olfaelamine@yahoo.fr

\begin{abstract}
Eccrine hidradenoma (EH) is a rare benign cutaneous neoplasm, believed to arise from the distal excretory duct of the eccrine sweat glands. We report an exceptional lesion of eccrine hidradenoma localized to the breast and clinically mimicking a carcinoma of the breast. Aim: We aim to describe the pathological characteristics of this lesion. We report the case of a 59 year old woman. She presented with a right-sided breast nodule. Physical examination, found a mobile firm nodule retracting the skin. By ultrasound examination, the tumor was heterogeneous and measured $11 \mathrm{~mm}$ in great diameter. A local surgical excision of the nodule was performed. By histological exam, the diagnosis of EH was retained. The complement of immunohistochemistry was not requested. This tumor is usually confined to the dermis or subcutaneous layer without. EH of the breast is an uncommon site. Histopathological confirmation is necessary and wide excision of these tumors is the treatment of choice.
\end{abstract}

Key words: Breast; Eccrine hidradenoma; Benign tumor

\section{INTRODUCTION}

Eccrine hidradenoma is a rare benign cutaneous neoplasm, believed to arise from the distal excretory duct of the eccrine sweat glands. Its histogenesis is not well elucidated, therefore eccrine hidradenoma is also known as nodular hidradenoma, clear cell hidradenoma $(\mathrm{CCH})$, eccrine acrospiroma, and eccrine sweat gland adenoma $[1,2]$. We report an exceptional lesion of eccrine hidradenoma localized to the breast and clinically mimicking a carcinoma of the breast. We aim to describe its pathological characteristics. Clinicians and pathologists should be aware of this lesion and do not confuse it with breast carcinomas.

\section{CASE REPORT}

We report the case of a female patient aged 59 years old with no particular disease history. She presented with a right-sided breast nodule dating of 6 months. Physical examination, found a mobile firm nodule retracting the overlying skin and measuring $10 \mathrm{~mm}$ in great diameter, sitting at the inferomedial inner quadrant. Left breast was normal, axillary and supraclavicular lymph nodes were free. Ultrasound examination, objectived an inferomedial right internal mammary nodule, measuring $11 \mathrm{~mm}$ large, heterogeneous and vascularized at the Doppler ultrasound examination. A local surgical excision of the nodule was performed. Grossly, we described a fragment measuring 2,5/1 cm, showing on cut section a well limited whitish firm nodule measuring $1 \mathrm{~cm}$. Histological examination showed a well limited dermal nodule, encapsulated and not connected to the epidermis (Fig. 1). It is represented by compact lobules. The tumor cells are polygonal with eosinophilic cytoplasm and non atypic nuclei (Fig. 2). Characteristically, we noted the presence of some cavities filled with lacquered appearance. Some lobules

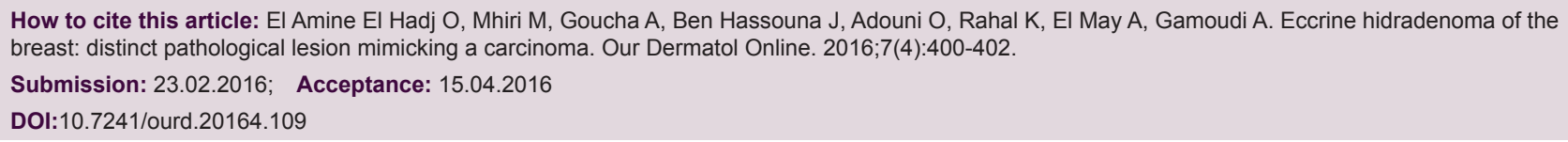




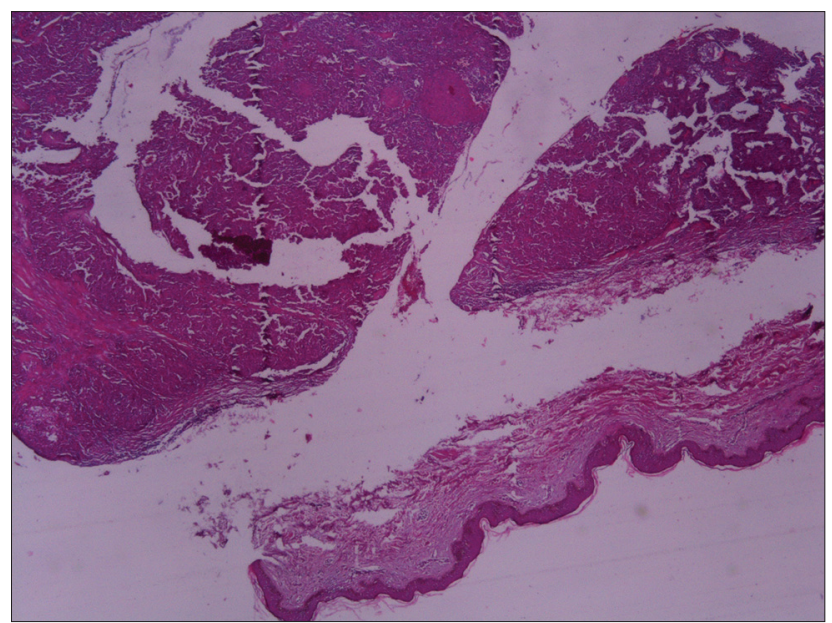

Figure 1: A well limited dermal nodule encapsulated and not connected to the epidermis.

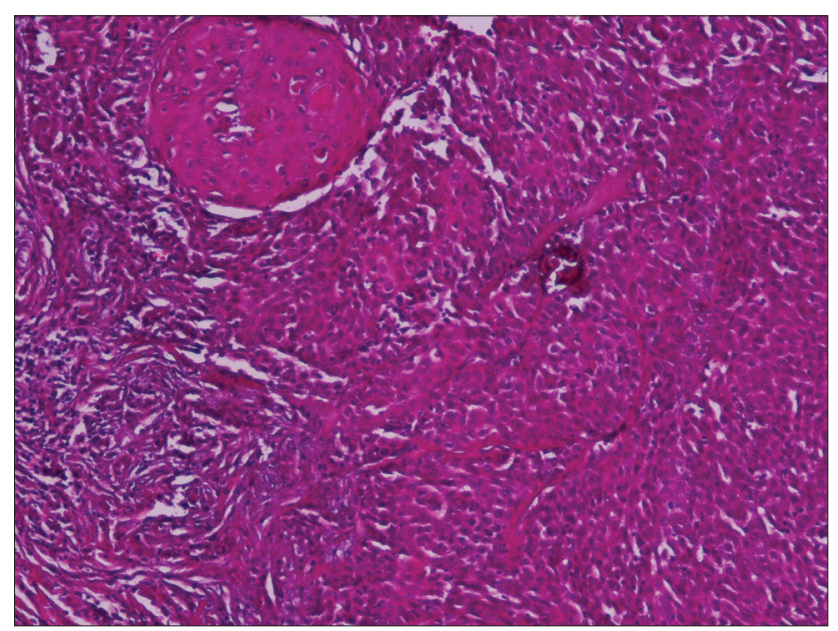

Figure 2: Tumor cells are polygonal with eosinophilic cytoplasm and non atypic nuclei.

showed squamous differenciation. The diagnosis of eccrine hidradenoma was retained. The complement of immunohistochemistry was not requested.

\section{DISCUSSION}

Eccrine hidradenoma's affected more frequently women than males [3]. It occurs commonly in middle-aged and older adults, and can be found anywhere on the body without predilection for a particular site [1]. However common reported sites of origin of $\mathrm{CCH}$ are face, upper extremity, axilla, trunk, thigh, the ear Lobule, scalp and pubic region [4]. Although several skin adnexal tumors arise in the breast tissue, $\mathrm{CCH}$ of the breast is an extremely rare entity [3,4]. It usually manifests as an asymptomatic, solitary, firm, cutaneous nodule, measuring between 5 and $20 \mathrm{~mm}$ in diameter. Several reports of giant eccrine hidradenoma can be found in the literature. The tumor is usually confined to the dermis or subcutaneous layer without involving the deep structures [1]. Its growth is slow, and a malignant change is rare [1].

Malignant transformation is extremely rare, and eccrine hidradenoma does not have a characteristic clinical appearance; therefore, differential diagnosis from other neoplasms, either benign or malignant, is only possible after a histopathological evaluation [1].

Usually, CCH is difficult to diagnose cytologically and in most studies, cytological diagno- sis was either unconclusive or misdiagnosed as breast tumor [4].

On histopathological examinations these tumours represent two types of cells, predominantly round cells with clear cytoplasm and polyhedral cells with rounded nucleus and slightly basophilic cytoplasm. The clear cells contain glycogen and Periodic Acid-Schiff-positive, diastase-resistant material, but no lipid. Focal cystic change also seen. Rarely some lesions show squamous differentiation which is observed in our case. Furthermore, malignant transformation is possible but extremely rare [3] and has been observed in only few cases [4]. However, neither clinical behavior nor histological features can predict malignant change. It can occur in case of inadequate excision. Compared to benign lesions malignant lesions has more tendencies to invade local tissue [3].

Although the exact mechanism of invasion by benign tumors has not yet been elucidated, benign tumor cells might invade into the deep structures along nerves such as the facial nerve and trigeminal nerve $[1,2]$. One report explained the mechanism of metastasis of benign tumors: the classical metastatic properties of malignant cells are the consequence of genetic abnormalities; conversely, benign metastasis can be explained by a mechanical "squeezing" of neoplastic cells into the lymph vessels after tumor overgrowth and/or trauma. Some autors speculate that the tumor cells might migrate along the lymphatics and/or by neurovascular permeation and forme a remote mass separated from the main tumor. Therefore, benign eccrine hidradenoma has a tendency to show features of a malignant tumor, such as regional metastasis and infiltration of the deep structures [1].

Immunohistochemistry (IHC) further aids in ruling other differentials. CCH shows reactivity for P63, 
keratin, EMA, CEA, S-100 and Vimetin but negativity for alpha-SMA, CD-10, ER, PR [4].

Complete surgical excision with safe margins and regular follow-up is the treatment of choice for breast CCH [4].

\section{CONCLUSION}

$\mathrm{CCH}$ should be considered in the differential diagnosis of breast neoplasm on cytology. Awareness of these lesions on cytology is essential to prevent misdiagnosis and over treatment [4]. Histopathologic confirmation is necessary and wide excision of tumour is the treatment of choice [2].

\section{Consent}

The examination of the patient was conducted according to the Declaration of Helsinki principles.
Written informed consent was obtained from the patient for publication of this article.

\section{REFERENCES}

1. Seung Hyun Oh, Chung Hun Kim, Euna Hwang. Giant Eccrine Hidradenoma Mimicking a Malignant Tumor of the Salivary Gland. Arch Surg Pathol. 2014;41):437-39.

2. Ohnishi I, Watanabe S. Histogenesis of clear cell hidradenoma: immunohistochemical study of keratin expression. J Culan Pathol. 1997;24:30-6.

3. Myreddy N, Pagatikaluva VR, Subbarayula ST, Gari SP, Gari BC. Clear Cell Hidradenoma of the Ear Lobule: An Unusual Site of Presentation. J Clin Diagn Res. 2015;9:ED20-1.

4. Sehgal S, Goyal P, Ghosh S, Mittal D, Kumar A, Singh S. Clear cell hidradenoma of breast mimicking atypical breast lesion: a diagnostic pitfall in breast cytology. Rare Tumors. 2014;6:5343. Rare Tumors 2014; volume 6:5343

Copyright by Olfa El Amine El Hadj, et al. This is an open access article distributed under the terms of the Creative Commons Attribution License, which permits unrestricted use, distribution, and reproduction in any

medium, provided the original author and source are credited.

Source of Support: Nil, Conflict of Interest: None declared. 